\title{
The Impact of Nuclear Physics Uncertainties on Interpret- ing Kilonova Light Curves
}

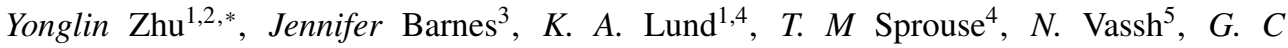 \\ McLaughlin $^{1,2}, M$. R Mumpower ${ }^{4,6}$, and $R$. Surman ${ }^{2,5}$ \\ ${ }^{1}$ Dept. of Physics, North Carolina State University, Raleigh NC 27695 USA, \\ ${ }^{2}$ Joint Institute for Nuclear Astrophysics - Center for the Evolution of the Elements, USA, \\ ${ }^{3}$ Dept. of Physics and Columbia Astrophysics Laboratory, Columbia University, NY 10027 USA, \\ ${ }^{4}$ Theoretical Division, Los Alamos National Laboratory, Los Alamos NM 87545 USA \\ ${ }^{5}$ Dept. of Physics, University of Notre Dame, Notre Dame IN 46556 USA, \\ ${ }^{6}$ Center for Theoretical Astrophysics, Los Alamos National Laboratory, Los Alamos, NM, 87545, USA
}

\begin{abstract}
Recently, analysis of the optical counterpart AT2017gfo to the gravitational wave-detected neutron star merger GW170817 has suggested a promising resolution of a long-standing debate on neutron star mergers as a source of some of the heaviest elements. However, making quantitative progress in these areas requires an accounting of the uncertainties in different aspects of physics, which is input into simulations of merging compact objects and their associated phenomena, remarkably rapid neutron capture nucleosynthesis. We investigate the uncertainties from the nuclear inputs to rapid neutron capture nucleosynthesis calculations combining different theoretical nuclear mass models, spontaneous fission rates, and fission daughter product distributions on top of the experimental nuclear data. We report that such nuclear physics uncertainties typically generate at least one order of magnitude uncertainty in the nuclear heating, which leads to uncertainties in the bolometric luminosity and the inferred mass of r-process material from the kilonova light curve.
\end{abstract}

\section{Introduction}

The detailed observations of GW170817 provides a definitive answer to the question of heavy element origin: binary neutron star mergers (NSMs) are a source of elements heavier than iron. Current simulations could fit optical counterpart to GW170817 qualitatively well; meanwhile, quantitative differences in simulations could be by orders of magnitude (see discussion in [1]). In order to accurately interpret kilonova emission and understand the evolution of $r-$ process elements in the Universe, recent studies has attempted to explore the complexity from astrophysics[2-4], nuclear physics[5-7] and atomic physics[8].

\footnotetext{
*e-mail: zhuygln@gmail.com
} 


\section{Objective}

We investigate uncertainties from the nuclear inputs to rapid neutron capture nucleosynthesis calculations with a focus on nuclear heating and the impact on kilonova light curves. We demonstrate how these nuclear physics uncertainties propagate to nuclear heating and inferred astrophysical quantities such as ejecta mass.

\section{Method}

We consider lanthanide-rich outflows to emphasize contributions from species with $\mathrm{A} \geq 120$ in the light curve, following setup and treatments from [5]. These lanthanide-rich outflows will be useful for elucidating neutron-rich ejecta from the mergers of binary neutron stars, as well as black hole-neutron star systems. We start with a base astrophysical trajectory of a standard parameterized wind from [9] and use the electron fraction $Y_{e}$ as a proxy for all astrophysical inputs. We generate seed nuclear abundance wtih SFHo equation of state (Steiner et al. 2012) and perform the nucleosynthesis simulations with the Portable Routines for Integrated nucleoSynthesis Modeling(PRISM). We explore the uncertainties from theoretical nuclear inputs in regions where experimental measured mass from AME2016 [10] and experimental data from NUBASE2016 [11] are not available. We adopt the treatment in thermalization efficiencies for effective heating from [12] and opacity from [13] as input into a semi-analytical light curve model. We selected 13 highlighted simulations as in Table 1 to cover reasonable electron fractions and popular nuclear physics inputs(nuclear mass models, fission prescriptions). In our kilonvoa light curve calculations, we use a single-component (red) model of a range of given mass and velocity of $v_{e j}=0.15 c$.

Table 1. Highlighted Simulations Setup (for details see [5])

\begin{tabular}{cccccc}
\hline \hline Index & Mass Model & $Y_{e}$ & Yield Distribution & Spontaneous Fission rates \\
\hline 1 & FRDM2012 & 0.28 & Symmetric & Karpov/Zagrebaev \\
2 & FRDM2012 & 0.16 & Symmetric & Karpov/Zagrebaev \\
3 & HFB22 & 0.16 & Symmetric & Karpov/Zagrebaev \\
4 & HFB27 & 0.16 & Kodama & Karpov/Zagrebaev \\
5 & DZ33 & 0.16 & Symmetric & Karpov/Zagrebaev & \\
6 & UNEDF1 & 0.16 & Kodama & Karpov/Zagrebaev & $a$ \\
7 & UNEDF1 & 0.16 & Kodama & Xu \& Ren & \\
8 & UNEDF1 & 0.24 & Symmetric & Karpov/Zagrebaev & \\
9 & SLY4 & 0.18 & Symmetric & Karpov/Zagrebaev \\
10 & SLY4 & 0.21 & Symmetric & Karpov/Zagrebaev \\
11 & TF+D3C* & 0.16 & Symmetric & Karpov/Zagrebaev \\
12 & DZ33 & mixture & \\
13 & UNEDF1 & mixture & Kodama & Symmetric & Karpov/Zagrebaev \\
\end{tabular}

${ }^{b}$ Linear combinations of trajectories with different $Y_{e}$ values that fit the solar r-process abundance.

\section{Results}

As can be seen in Figure 7 in [5], the final abundances from the selected simulations differ in the abundance of r-process elements by two to three orders of magnitude. Relevant to the opacity calculations used in kilonova light curve simulations, the mass fraction of lanthanides 
and actinides at one day after merger differs from $10^{-2}$ to more than $10^{-1}$ as shown in subbarplot of Figure 1. We use this to explore the light curve with a grid of ejecta mass values for these 13 models as colored and grey solid lines in Figure 1, comparing with the observed values (diamond markers). We highlight one light curve for each of the 13 simulations that best matches the late time observation from GW170817 with color and find that their ejecta masses range from $0.01 M_{\odot}$ to $0.08 M_{\odot}$. We different power law fits to different light curves - for simulations with more contribution from fission and/or $\alpha$-decay, such as simulation 3 in blue line, the light curves follow a slower decay rate like $L \propto t^{-1}$; while simulations that are not neutron-rich enough to make heavy nuclei that undergo fission and $\alpha$-decay, such as simulation 1 , follow a faster decay rate as $L \propto t^{-2.7}$.

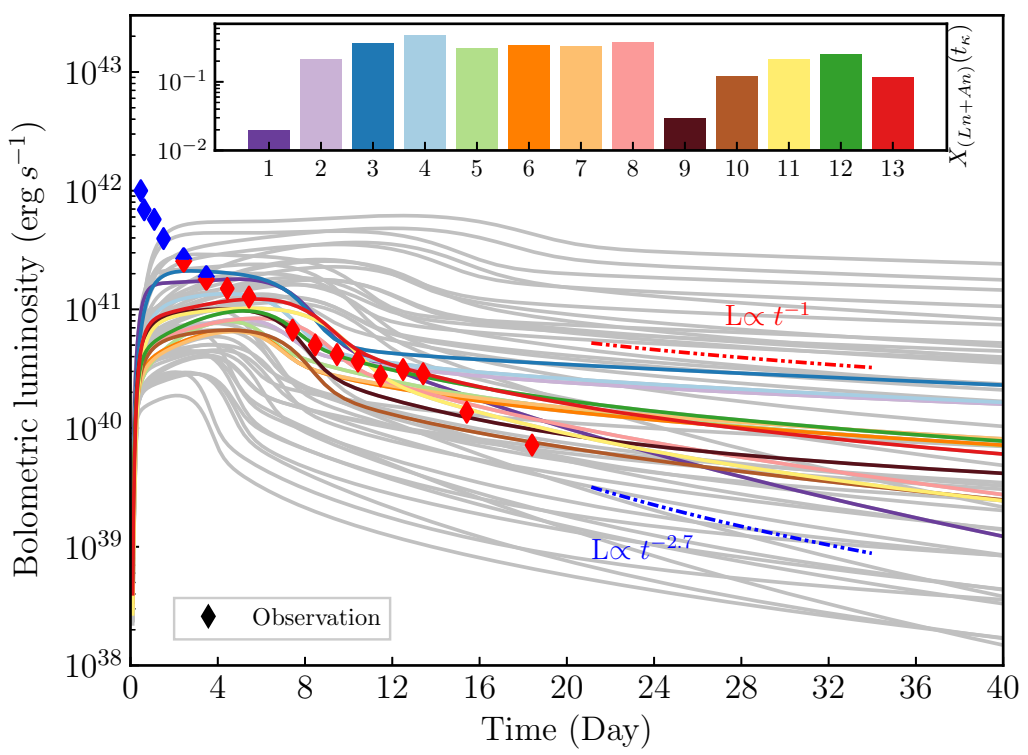

Figure 1. Bolometric luminosity of selected simulations with different eject masses. The subplot in the top is mass fractions of lanthanides $(L n)$ and actinides $(A n), X_{\mathrm{Ln}+\mathrm{An}}$ at one day after merger. Blue and red diamond markers are observed bolometric luminosity. Dash-doted lines with labels of $L \propto t^{-\alpha}$ show power fit to the bolometric luminosity. The colored lines show light curve simulations that are closest to the AT2017gfo observation of the inferred ejecta masses grid. The inferred ejecta masses used for each colored lines are $0.05 M_{\odot}, 0.02 M_{\odot}, 0.01 M_{\odot}, 0.01 M_{\odot}, 0.01 M_{\odot}, 0.02 M_{\odot}, 0.02 M_{\odot}, 0.05 M_{\odot}, 0.08 M_{\odot}$, $0.08 M_{\odot}, 0.02 M_{\odot}, 0.05 M_{\odot}$ by order. The grey lines shows the light curves with the rest of ejecta mass grid for each simulations. This figure is made from same calculations as in figure 8 and 12 from [5].

\section{Conclusion}

For lanthanide-rich outflows, nuclear inputs introduce uncertainty in lanthanide and actinide production to at least one order of magnitude around one day after merger. We show that the uncertainty in effective nuclear heating propagates to bolometric luminosity with the same order of magnitude from days to hundreds of days after merger. We find that power fit may be useful to estimate light curve at a different times with consideration of dominating re- 
action channels. We demonstrate that the inferred quantities like mass of ejecta could be significantly impacted by the choice of nuclear physics inputs in the simulations.

\section{References}

[1] A. Aprahamian, R. Surman, A. Frebel, G. C. McLaughlin, A. Arcones, A. B. Balantekin, J. Barnes, T. C. Beers, E. M. Holmbeck and J. Yoon, et al. [arXiv:1809.00703 [astroph.HE]].

[2] O. Korobkin, R. Wollaeger, C. Fryer, A. L. Hungerford, S. Rosswog, C. Fontes, M. Mumpower, E. Chase, W. Even and J. M. Miller, et al. Astrophys. J. 910, no.2, 116 (2021) doi:10.3847/1538-4357/abe1b5 [arXiv:2004.00102 [astro-ph.HE]].

[3] F. Foucart, P. Moesta, T. Ramirez, A. J. Wright, S. Darbha and D. Kasen, [arXiv:2109.00565 [astro-ph.HE]].

[4] R. T. Wollaeger, C. L. Fryer, E. A. Chase, C. J. Fontes, M. Ristic, A. L. Hungerford, O. Korobkin, R. O'Shaughnessy and A. M. Herring, Astrophys. J. 918, no.1, 10 (2021) doi:10.3847/1538-4357/ac0d03 [arXiv:2105.11543 [astro-ph.HE]].

[5] Y. L. Zhu, K. Lund, J. Barnes, T. M. Sprouse, N. Vassh, G. C. McLaughlin, M. R. Mumpower and R. Surman, Astrophys. J. 906 (2021) no.2, 94 doi:10.3847/15384357/abc69e [arXiv:2010.03668 [astro-ph.HE]].

[6] J. Barnes, Y. L. Zhu, K. A. Lund, T. M. Sprouse, N. Vassh, G. C. McLaughlin, M. R. Mumpower and R. Surman, Astrophys. J. 918, no.2, 44 (2021) doi:10.3847/15384357/ac0aec [arXiv:2010.11182 [astro-ph.HE]].

[7] Y. L. Zhu, T. Sprouse, M. R. Mumpower, N. Vassh, R. Surman and G. C. McLaughlin, Springer Proc. Phys. 219, 469-472 (2019) doi:10.1007/978-3-030-13876-9_92

[8] K. Hotokezaka, M. Tanaka, D. Kato and G. Gaigalas, doi:10.1093/mnras/stab1975 [arXiv:2102.07879 [astro-ph.HE]].

[9] I. V. Panov and H. T. Janka, Astron. Astrophys. 494, 829 (2009) doi:10.1051/00046361:200810292 [arXiv:0805.1848 [astro-ph]].

[10] M. Wang, W. J. Huang, F. G. Kondev, G. Audi and S. Naimi, Chin. Phys. C 45, no.3, 030003 (2021) doi:10.1088/1674-1137/abddaf

[11] G. Audi, F. G. Kondev, M. Wang, W. J. Huang and S. Naimi, Chin. Phys. C 41, no.3, 030001 (2017) doi:10.1088/1674-1137/41/3/030001

[12] D. Kasen and J. Barnes, Astrophys. J. 876, no.2, 128 (2019) doi:10.3847/15384357/ab06c2 [arXiv:1807.03319 [astro-ph.HE]].

[13] D. Kasen, N. R. Badnell and J. Barnes, Astrophys. J. 774, 25 (2013) doi:10.1088/0004637X/774/1/25 [arXiv:1303.5788 [astro-ph.HE]]. 